\title{
The Multivariate Saddlepoint Approximation to the Distribution of Estimators: A General Approach
}

\author{
Juan Carlos Abril, María de las Mercedes Abril and Carlos I. Martinez \\ Universidad Nacional de Tucumán and Consejo Nacional de Investigaciones Cientificas y Técnicas (CONICET). Tucumán, Argentina.
}

Received: November 02, 2015 / Accepted: November 30, 2015 / Published: February 25, 2016.

\begin{abstract}
We develop the theory of multivariate saddlepoint approximations. Our treatment differs from the one in Barndorff-Nielsen and Cox (1979, equation (4.7)) in two aspects: 1) our results are satisfied for random vectors that are not necessarily sums of independent and identically distributed random vectors, and 2) we consider that the sample is taken from any distribution, not necessarily a member of the exponential family of densities. We also show the relationship with the corresponding multivariate Edgeworth approximations whose general treatment was developed by Durbin in 1980, emphasizing that the basic assumptions that support the validity of both approaches are essentially similar.
\end{abstract}

Key words: Approximate distributions, Asymptotic expansions, Edgeworth approximations, Saddlepoint approximations.

\section{Introduction}

The main purpose of this paper is to present the theory that sustains multivariate saddlepoint expansions. These were introduced in statistics in a pioneering work of Daniels (1954) for the univariate case. Barndorff-Nielsen and Cox (1979) studied the multivariate case, but basically their focus is centred on applications to sums of independent and identically distributed vectors and when the underlying distributions are members of the exponential family.

Let $\mathbf{y}=\left(\mathbf{y}_{1}, \ldots, \mathbf{y}_{n}\right)^{\prime}$ be a matrix of observations of $n$ continuous or discrete random $l \times 1$ vectors, not necessarily independent or identically distributed with a density given by $f(\mathbf{y}, \boldsymbol{\theta})$ where the parameter vector $\boldsymbol{\theta}$ is $q$-dimensional, $\boldsymbol{\theta} \in \Theta$, and let $\boldsymbol{\theta}_{0}$ be the true value of the parameter vector $\boldsymbol{\theta}$.

Suppose that the $m$-dimensional random vector

$$
\mathbf{S}_{n}=\mathbf{S}_{n}(\mathbf{y})=\left(\begin{array}{llll}
S_{1 n}, & S_{2 n} & \ldots & S_{m n}
\end{array}\right)^{\prime}
$$

Corresponding author: Juan Carlos Abril, Universidad Nacional de Tucumán and Consejo Nacional de Investigaciones Científicas y Técnicas (CONICET). Tucumán, Argentina. E-mail: jabril@herrera.unt.edu.ar. has a density with respect to Lebesgue measure which depends on integer $n>N$ for some positive $N$ and on $\boldsymbol{\theta} \in \Theta$, where $\Theta$ is a subset of $\mathfrak{R}^{q}$ for $q$ an arbitrary positive integer.

Let

$$
\phi_{n}(\mathbf{z})=E\left(e^{i \mathbf{z}^{\prime} \mathbf{S}_{n}}\right)
$$

be the characteristic function of $\mathbf{S}_{n}$, where $i$ is the imaginary unit and $\mathbf{z}=\left(z_{1}, z_{2}, \ldots, z_{m}\right)^{\prime}$. The cumulant generating function of $\mathbf{S}_{n}$ is

$$
\psi_{n}(\mathbf{z})=\log \phi_{n}(\mathbf{z}) .
$$

Whenever the appropriate derivatives exist, let $\partial^{j} \psi_{n}(\widetilde{\mathbf{z}}) / \partial \mathbf{z}^{j}$ denote the set of $j$ th order derivatives

$$
\frac{\partial^{j} \psi_{n}(\widetilde{\mathbf{z}})}{\partial z_{1}^{j_{1}} \ldots \partial z_{m}^{j_{m}}},
$$

for all integers $j_{1}, \ldots, j_{m} \geq 0$ satisfying $\sum_{k} j_{k}=j$, evaluated at $\mathbf{z}=\widetilde{\mathbf{z}}$. The $j$ th cumulant $\kappa_{n j}$ of $\mathbf{S}_{n}$ when it exists, satisfies the relation

$$
i^{j} \kappa_{n j}=\frac{\partial^{j} \psi_{n}(\mathbf{0})}{\partial \mathbf{z}^{j}} .
$$

It is important to note that if $\mathbf{S}_{n}$ is of dimension $m>1$ then $\kappa_{i j}$ is of dimension greater than 1 for all 
$j$. Only when $m=1$ the dimension of the cumulants defined in (5) is 1 . Note that $\phi_{n}$ and $\psi_{n}$ depend on the parameter vector $\boldsymbol{\theta}$ but we will not write it explicitly to keep the notation as simple as possible.

Let $h_{n}(\mathbf{x}, \boldsymbol{\theta})$ be the density of the random vector

$$
\mathbf{X}_{n}=n^{-1 / 2}\left\{\mathbf{S}_{n}-E\left(\mathbf{S}_{n}\right)\right\} .
$$

We will derive, and bound the error of, the multivariate saddlepoint approximations to the density $h_{n}(\mathbf{x}, \boldsymbol{\theta})$. Our treatment differs from Barndorff-Nielsen and Cox (1979) in two aspects: 1) our results are satisfied for random vectors that are not necessarily sums of independent and identically distributed random vectors, and 2) we consider that the sample is taken from any distribution, not necessarily a member of the exponential family of densities.

We also show the relationship with the corresponding multivariate Edgeworth approximations whose general treatment was developed by Durbin (1980), emphasizing that the basic assumptions that support the validity of both approaches are essentially similar. Finally, we present an example and perform an analysis of the results through comparisons between Monte Carlo simulations and the approximations we obtained.

\section{Multivariate Saddlepoint Expansions}

Just as we stated before, the main purpose of this paper is to present the theory of multivariate saddlepoint expansions. These were introduced in statistics in a pioneering work of Daniels (1954) for the univariate case. Barndorff-Nielsen and Cox (1979) studied the multivariate case, but basically his focus is centred on applications to sums of independent and identically distributed vectors and when the underlying distributions are members of the exponential family.

The characteristic function of $\mathbf{X}_{n}$ is

$$
\phi_{\mathbf{X}_{n}}(\mathbf{z})=e^{-i \frac{\mathbf{z}^{\prime}}{\sqrt{n}} E\left(\mathbf{S}_{n}\right)} \phi_{n}\left(\frac{\mathbf{z}}{\sqrt{n}}\right),
$$

where $\phi_{n}$ is the characteristic function of $\mathbf{S}_{n}$, and the corresponding cumulant generating function is

$$
\psi_{\mathbf{X}_{n}}(\mathbf{z})=\log \phi_{\mathbf{X}_{n}}(\mathbf{z})=\psi_{n}\left(\frac{\mathbf{z}}{\sqrt{n}}\right)-i \frac{\mathbf{z}^{\prime}}{\sqrt{n}} E\left(\mathbf{S}_{n}\right) .
$$

We define the operator $\nabla=\left(\frac{\partial}{\partial z_{1}}, \ldots, \frac{\partial}{\partial z_{m}}\right)^{\prime}$, and where $\nabla^{k} \psi_{X_{n}}(\widetilde{\mathbf{z}})$ means that we evaluate at $\mathbf{z}=\widetilde{\mathbf{z}}$ the $k$ th derivatives of $\psi_{\mathbf{X}_{n}}(\mathbf{z})$ with respect to the elements of the vector $\mathbf{z}$ for $k=1,2, \ldots$.

Let $\hat{\mathbf{z}}=\left(\hat{z}_{1}, \hat{z}_{2}, \ldots, \hat{z}_{m}\right)^{\prime}$ be the root of the equation

$$
\nabla\left\{\psi_{X_{n}}(\mathbf{z})-i \mathbf{z}^{\prime} \mathbf{x}\right\}=\mathbf{0},
$$

which is the solution of

$$
\frac{\partial \psi_{\mathbf{x}_{n}}(\mathbf{z})}{\partial \mathbf{z}}=i \mathbf{x} .
$$

In what follows we need to introduce the following three assumptions:

Assumption 1: If $n$ is large enough $\left|\phi_{n}(\mathbf{z})\right|$ is integrable over $\mathfrak{R}^{m}$, and if $\delta_{1}$ is an arbitrary positive constant the limit of

$$
n^{\frac{r}{2}-1} \int_{B_{\delta_{1}} \sqrt{n}} \mid \phi_{n}(\mathbf{z} / \sqrt{n}) d \mathbf{z}
$$

as $n \rightarrow \infty, \quad \boldsymbol{\theta} \rightarrow \boldsymbol{\theta}_{0}$, is zero, where $B_{\delta_{1} \sqrt{n}}$ is the region that contains $\hat{\mathbf{z}}$ of (10) and that $\|\mathbf{z}\|>\delta_{1} \sqrt{n}$ where \|\| denotes the Euclidean norm.

Assumption 2: The derivatives

$$
\frac{\partial^{j} \psi_{n}(\mathbf{z})}{\partial \mathbf{z}^{j}}
$$

exist for $\mathbf{z}$ in neighborhoods of the origin and of $\hat{\mathbf{z}}$ given in (9), for $\boldsymbol{\theta}$ in a neighborhood of $\boldsymbol{\theta}_{0}$ and for $j=1, \ldots, r$. Also, the limit of $n^{-1} \frac{\partial^{r} \psi_{n}(\mathbf{z})}{\partial \mathbf{z}^{r}}$ exists when $n \rightarrow \infty, \quad \boldsymbol{\theta} \rightarrow \boldsymbol{\theta}_{0}$ in neighborhoods of the origin and of $\hat{\mathbf{z}}$ given in (9).

Assumption 3: The derivatives 


$$
\frac{\partial^{j} \psi_{n}(\mathbf{z})}{\partial \mathbf{z}^{j}}=O(n)
$$

uniformly for $\boldsymbol{\theta}$ in a neighborhood of $\boldsymbol{\theta}_{0}$; for $\mathbf{z}$ in neighborhoods of the origin and of $\hat{\mathbf{z}}$ given in (9) and for $j=1, \ldots, r-1$.

Note that assumptions 1, 2 and 3 above were placed so that they contain assumptions 2, 3 and 4 given in Durbin (1980a) in his general treatment of multivariate Edgeworth expansions. In effect, taking $\hat{\mathbf{z}}$ not as root of the equation (10) but equal to the null vector $\mathbf{0}$, everything leads us to what was discussed by Durbin (1980a).

Theorem 1: Suppose that there is an integer $r \geq 4$ such that assumptions 1,2 and 3 hold, then it is possible to obtain the saddlepoint approximation

$$
\hat{h}_{n}(\mathbf{x}, \boldsymbol{\theta})=\frac{1}{(2 \pi)^{m / 2}}|\mathbf{B}|^{-1 / 2} \exp \left\{\psi_{\mathbf{x} n}(\hat{\mathbf{z}})-i \hat{\mathbf{z}}^{\prime} \mathbf{x}\right\},
$$

to the density $h_{n}(\mathbf{x}, \boldsymbol{\theta})$ of the random vector $\mathbf{X}_{n}$, uniformly in $\mathbf{x}$ and in $\boldsymbol{\theta}$ for a neighborhood $\left\|\boldsymbol{\theta}-\boldsymbol{\theta}_{0}\right\|<\delta_{2}$ where $\delta_{2}$ is a is a suitably chosen positive constant independent of $n$ and

$$
\mathbf{B}=-\frac{\partial^{2} \psi_{\mathbf{X} n}(\hat{\mathbf{z}})}{\partial \mathbf{z} \partial \mathbf{z}} \text {. }
$$

The proof can be sent on request.

\section{Error Bound of the Multivariate Saddlepoint Approximation}

Let us take, not the density $h_{n}(\mathbf{x}, \boldsymbol{\theta})$ but a proper member of the exponential family or the conjugate family as stated in Kinchin (1949)

$$
f_{n}(\mathbf{x}, \boldsymbol{\theta}, \boldsymbol{\lambda})=\frac{e^{i \lambda^{\prime} \mathbf{x}} h_{n}(\mathbf{x}, \boldsymbol{\theta})}{\phi_{\mathbf{x}_{\mathbf{n}}}(\boldsymbol{\lambda})},
$$

where the function $\phi_{\mathbf{x}_{n}}$ was defined in (7). Clearly, $h_{n}(\mathbf{x}, \boldsymbol{\theta})$ depends on the parameter vector $\boldsymbol{\theta}$ of dimension $q$ and the density function $f_{n}(\mathbf{x}, \boldsymbol{\theta}, \boldsymbol{\lambda})$ depends on the parameter $\boldsymbol{\gamma}=(\boldsymbol{\theta}, \boldsymbol{\lambda})^{\prime}$ of dimension $q+n$.

Formula (16) can be written in the more familiar form

$$
f_{n}(\mathbf{x}, \boldsymbol{\theta}, \boldsymbol{\lambda})=\exp \left\{i \mathbf{z}^{\prime} \mathbf{x}-\alpha(\mathbf{x}, \boldsymbol{\theta})-\beta(\boldsymbol{\lambda})\right\}
$$

It is important to see that when $\boldsymbol{\lambda}=\mathbf{0}$ then $f(\mathbf{x}, \boldsymbol{\theta}, \boldsymbol{\lambda}, \mathbf{0})=h_{n}(\mathbf{x}, \boldsymbol{\theta})$.

Suppose that we are interested in the density $f_{n}\left(\mathbf{x}, \boldsymbol{\theta}, \boldsymbol{\lambda}_{0}\right)$ for some particular value $\boldsymbol{\lambda}_{0}$ of $\boldsymbol{\lambda}$. Then, for any $\lambda$ we have that

$$
\begin{aligned}
f_{n}\left(\mathbf{x}, \boldsymbol{\theta}, \boldsymbol{\lambda}_{0}\right) & =\exp \left\{\beta(\boldsymbol{\lambda})-\beta\left(\boldsymbol{\lambda}_{0}\right)+i\left(\boldsymbol{\lambda}-\boldsymbol{\lambda}_{0}\right)^{\prime} \mathbf{x}\right\} f_{n}(\mathbf{x}, \boldsymbol{\theta}, \boldsymbol{\lambda}) \\
& =\exp \left\{\psi_{\mathbf{x}_{n}}(\boldsymbol{\lambda})-\psi_{\mathbf{x}_{n}}\left(\boldsymbol{\lambda}_{0}\right)+i\left(\boldsymbol{\lambda}-\boldsymbol{\lambda}_{0}\right)^{\prime} \mathbf{x}\right\} \\
& \times f_{n}(\mathbf{x}, \boldsymbol{\theta}, \boldsymbol{\lambda})
\end{aligned}
$$

Therefore, an approximation to $f_{n}\left(\mathbf{x}, \boldsymbol{\theta}, \boldsymbol{\lambda}_{0}\right)$ can be obtained via an approximation based on the Edgeworth expansion of $f_{n}(\mathbf{x}, \boldsymbol{\theta}, \boldsymbol{\lambda})$ for any $\boldsymbol{\lambda}$ satisfying the assumptions made in Theorem 1 of Durbin (1980).

On the other hand, when we consider $\boldsymbol{\lambda}_{0}=\mathbf{0}$ and $\lambda=\hat{\mathbf{z}}$ we may see that

$$
\begin{aligned}
f_{n}(\mathbf{x}, \boldsymbol{\theta}, \boldsymbol{0}) & =h_{n}(\mathbf{x}, \boldsymbol{\theta}) \\
& =\exp \left\{\psi_{\mathbf{x}_{n}}(\hat{\mathbf{z}})-i \hat{\mathbf{z}}^{\prime} \mathbf{x}\right\} f_{n}(\mathbf{x}, \boldsymbol{\theta}, \hat{\mathbf{z}}) .
\end{aligned}
$$

In this formula, if we replace $f_{n}(\mathbf{x}, \boldsymbol{\theta}, \hat{\mathbf{z}})$ on the right hand side for the corresponding Edgeworth expansion, the result will lead to the saddlepoint approximation and its corresponding error bound of approximation of the density $h_{n}(\mathbf{x}, \boldsymbol{\theta})$. This is stated on the following theorem.

Theorem 2: Suppose that there is an integer $r=4$ such that assumptions 1, 2 and 3 stated above hold. Then there is a neighbourhood $\left\|\boldsymbol{\theta}-\boldsymbol{\theta}_{0}\right\|<\delta_{2}$ of $\boldsymbol{\theta}_{0}$ such that

$$
h_{n}(\mathbf{x}, \boldsymbol{\theta})=\hat{h}_{n}(\mathbf{x}, \boldsymbol{\theta})\left\{1+O\left(n^{-1}\right)\right\},
$$

uniformly in $\mathbf{x}$ and in $\boldsymbol{\theta}$ for $\left\|\boldsymbol{\theta}-\boldsymbol{\theta}_{0}\right\|<\delta_{2}$ where $\delta_{2}$ is a suitably chosen positive constant independent of $n$ and $\hat{h}_{n}(\mathbf{x}, \boldsymbol{\theta})$ is the saddlepoint approximation to the density $h_{n}(\mathbf{x}, \boldsymbol{\theta})$ of the random vector $\mathbf{X}_{n}$ defined in (14).

The proof can be sent on request. 


\section{Approximation to the Distribution of Estimators}

Usually one is interested in the saddlepoint approximation $\hat{g}(\mathbf{t}, \boldsymbol{\theta})$ to the distribution $g(\mathbf{t}, \boldsymbol{\theta})$ of the estimator $\mathbf{T}_{n}=n^{-1} \mathbf{S}_{n}$ for the parameter vector $\boldsymbol{\theta}$, where $\mathbf{S}_{n}$ satisfies assumptions 1,2 and 3 that we made before. What we may see is that maximum likelihood estimators often satisfy these assumptions. Using (6) we have in these cases $\mathbf{X}_{n}=n^{1 / 2}\left\{\mathbf{T}_{n}-E\left(\mathbf{T}_{n}\right)\right\}$.

Transforming from $\mathbf{x}$ to $\mathbf{t}$ in (14) and (19) we have

$$
\begin{aligned}
g(\mathbf{t}, \boldsymbol{\theta})= & \left(\frac{n}{2 \pi}\right)^{m / 2}|\mathbf{B}|^{-1 / 2} \\
& \times \exp \left\{\psi_{\mathbf{X}_{n}}(\hat{\mathbf{z}})-i n^{-1 / 2} \hat{\mathbf{z}}^{\prime}\left\{\mathbf{t}-E\left(\mathbf{T}_{n}\right)\right\}\right\} \\
& \times\left\{1+O\left(n^{-1}\right)\right\} \\
= & \hat{g}(\mathbf{t}, \boldsymbol{\theta})\left\{1+O\left(n^{-1}\right)\right\},
\end{aligned}
$$

uniformly in $\mathbf{t}$ for $\left\|\boldsymbol{\theta}-\boldsymbol{\theta}_{0}\right\|<\delta_{2}$, with $\hat{g}(\mathbf{t}, \boldsymbol{\theta})$ equal to the first two lines of (20)

\subsection{Integration over the Sample Space}

Of course, the establishment of an error bound on the density is not in itself of much direct practical use. What is needed for applications is a bound on the error of an integral over an appropriate region. The fact that the error term in (20) is a proportional error which is uniform in $\mathbf{t}$ for $\left\|\boldsymbol{\theta}-\boldsymbol{\theta}_{0}\right\|<\delta_{2}$, enables us to establish such a bound quite simple. The proof can be sent on request.

\subsection{Renormalization}

Daniels (1956) pointed out that when the constant term in the saddlepoint approximation is adjusted to make the integral over the whole space equal to unity, the order of magnitude of the error is often reduced from $n^{-1}$ to $n^{-3 / 2}$. He called this process renormalization. We show that the same result holds for the case of (20) and we obtain the following result

$$
g(\mathbf{t}, \boldsymbol{\theta})=\tilde{g}(\mathbf{t}, \boldsymbol{\theta})\left\{1+O_{x}\left(n^{-3 / 2}\right)\right\},
$$

where $\tilde{g}(\mathbf{t}, \boldsymbol{\theta})=K_{n}(\boldsymbol{\theta}) \hat{g}(\mathbf{t}, \boldsymbol{\theta})$ is the renormalized density and $K_{n}(\boldsymbol{\theta})$ comes from the following expression

$$
K_{n}^{-1}=\int \hat{g}(\mathbf{t}, \boldsymbol{\theta}) d \mathbf{t},
$$

where the integral is over the space $\mathfrak{R}^{m}$. If we work on (21) we will find out that the error term is not uniform in $\mathbf{x}$. Here we use the we use the notation $O\left(n^{-q}\right)$ introduced by Durbin(1980) to denote a quantity which $O\left(n^{-q}\right)$ for each fixed $\mathbf{x}$, but which is not $O\left(n^{-q}\right)$ uniformly for all $\mathbf{x}$.

What we finally see is that if $\mathbf{x}$ is held fixed, then $\tilde{g}(\mathbf{t}, \boldsymbol{\theta})$ has a proportional error of order $n^{-3 / 2}$. The proof can be sent on request.

\section{Example}

The aim of this example is to study an application of the saddlepoint approximations to non stationary $A R(1)$ models. Particularly, we want to investigate the saddlepoint approximations to the distribution of the estimator of the coefficient in non stationary $A R(1)$ models. In this case we study the approximations to the distribution of a univariate estimator allowing the analysis of the results through comparisons between Monte Carlo simulations and the approximations obtained.

We start with the model

$$
y_{t}=\rho y_{t-1}+\varepsilon_{t}, \quad t=1,2, \ldots, n,
$$

where $y_{0}=0$, the variables $\varepsilon_{1}, \varepsilon_{2}, \ldots, \varepsilon_{n}$ are independent and identical distributed $N\left(0, \sigma^{2}\right)$, $|\rho| \leq 1$ and we study approximations to the distribution of the estimator of the coefficient $\rho$. Firstly we develop the general case valid for all $\rho$ that is $|\rho| \leq 1$ and then we focus on the non stationary case under a unit root, when $\rho=1$. The results of this example are based on the work made in Abril, Abril and Martinez (2008). 
The sample, lag one, autocorrelation coefficient or serial correlation coefficient $r$ is usually used to estimate $\rho$ and is defined by

$$
r=\frac{\sum_{t=2}^{n} y_{t} y_{t-1}}{\sum_{t=1}^{n} y_{t}^{2}}=\frac{c_{1}}{c_{0}} .
$$

Using the results previously given, it is possible to show that

$$
\begin{aligned}
g(r, \rho) & =\frac{1}{2^{n+1} B\left(\frac{n+2}{2}, \frac{n+2}{2}\right)} \\
& \frac{\left(1-r^{2}\right)^{n / 2}}{(1-\rho r)\left(1-2 \rho r+\rho^{2}\right)^{(n-1) / 2}}\left\{1+O_{x}\left(n^{-3 / 2}\right)\right\},
\end{aligned}
$$

for $-1 \leq r \leq 1$, where $B(p, q)$ is a beta function defined as

$$
B(p, q)=\int_{0}^{1} u^{p-1}(1-u)^{q-1} d u=\frac{\Gamma(p) \Gamma(q)}{\Gamma(p+q)} .
$$

The proof can be sent on request.

In everything we previously did we have taken as a possible range of variation of the coefficient $\rho$ in the $A R(1)$ model to be $|\rho| \leq 1$ Therefore, making the corresponding transformations, we have that the second order approximation to the density of $r$ when $\rho=1$ is given by

$$
\begin{aligned}
g(r, 1) & =\frac{1}{2^{n+1} B\left(\frac{n+2}{2}, \frac{1}{2}\right)}(1-r)^{-1 / 2}(1+r)^{n / 2} \\
& \times\left\{1+O_{x}\left(n^{-3 / 2}\right)\right\},
\end{aligned}
$$

This last formula is our main result. Abril (2007) and Abril, Abril and Martinez (2007) studied the same problem applying Durbin's (1980a) method to obtain an approximation of order $n^{-3 / 2}$ to the density of $r$ when $\rho=1$ and they reach to a similar result but in that case $n$ is replaced by $n-1$.

The dominant term of (25) is a function that can be adequately integrated to obtain the significant points when we supposed that we have a process with a unit root.

Most of the available results in time series analysis, and particularly those related to $A R(1)$ models and unit roots are asymptotic. This means for the distributions of the different statistics of interest that they have a general error of approximation of order $n^{-1 / 2}$. For the model in (22) some results established by other authors improved this error taking it to one of order $n^{-1}$. Those improvements were made by the methods of Durbin and Edgeworth. The first of these methods is easily applicable and, as we pointed out earlier, produces similar formulas to those we obtained in this paper whereas the second method can give negative values on the tails of the distribution for this order of approximation.

The application of the saddlepoint approximation method to obtain a third order approximation to the density of $r$ when $\rho=1$ in (22) gives (25) as a result, which is similar to the corresponding formula obtained after applying the method of Durbin (1980a), but in this last case $n$ is replaced by $n-1$.

Formula (25) can be integrated for different samples sizes, or $n$. We can obtain values of $r$ that accumulate the $5 \%$ of the distribution. This can be interpreted as the $5 \%$ significant points of the distribution of the test statistic for the null hypothesis $\rho=1$ versus the alternative that is less than one. Comparing these with the results obtained using the method of Durbin, which can be seen in Abril, Abril and Martinez (2007), we can see that the $r$ values that accumulate the $5 \%$ of the area of the approximate distribution for the saddlepoint approximation method are always greater than the ones for the method of Durbin but these differences become smaller when $n$ increases, until it reaches values of $n$ equal to or greater that 100 , when it is practically zero. We note that in this case we do not studied the power of the possible test for the null hypothesis that $\rho=1$ versus the alternative that is less than one because our main objective is not to develop a statistic to test the presence of a unit root in (22) but to study second and third order saddlepoint approximations to the distribution of the estimator of 
the coefficient $\rho$, particularly when $\rho=1$.

Moreover, through simulations we have obtained the cumulative distribution of the estimator defined in (23). For this, we made 1000 replications and samples of different sizes have been taken, all under the assumption that $\rho=1$. To see the effect of changes in the number of replications we tested it with other values and found that it does not have significant influence on the results achieved, thus we decided to work with 1000 . The simulation results were compared with those achieved by the approximation given in (25) for the respective sample sizes.

The results obtained in this example for the model in (22) are better than those achieved previously by other researchers, mainly because they are not so complicated, as it is usually assumed for the saddlepoint methods and their interpretation is easy. Indeed, the basic approximation is closely related to the variance ratio distribution. In addition, we have improved the order of magnitude of the error taking it to $O\left(n^{-3 / 2}\right)$. But as (25) is an approximating formula, this means that it has differences with the formula of the exact distribution of the estimator, which we do not know. These differences were studied by simulations, as stated before, and from the results obtained, we can conclude that the approximation in (25) works well for the case of a non-stationary $A R(1)$ with $\rho=1$.

\section{References}

[1] Abril, J. C. (1984). Un estudio de las aproximaciones a las densidades con énfasis en las expansiones de Edgeworth. Estadistica - Journal of the Inter-American Statistical Institute, 36, 109-24.

[2] Abril, J.C. (1985). Asymptotic expansions for time series problems with applications to moving average models. Ph.D. Thesis, The London School of Economics and Political Science, University of London, England.

[3] Abril, J.C. (1987). The approximate densities of some quadratic forms of stationary random variables. Journal of Time Series Analysis. 8, 249-59.

[4] Abril, J.C. (2004). Modelos para el Análisis de las Series de Tiempo. Ediciones Cooperativas, Buenos Aires.
[5] Abril, J. C., Abril, M. de las M. and Martínez, C. I. (2007). Aproximaciones a la distribución del estimador del coeficiente en modelos AR(1) no estacionarios. Estadistica - Journal of the Inter-American Statistical Institute, 59, 87-102.

[6] Abril, J. C., Abril, M. de las M. and Martínez, C. I. (2008). La aproximación de punto de ensilladura a la distribución del coeficiente de correlación serial en el caso de raíz unitaria. Actas del VIII CLATSE (Congreso Latinoamericano de Sociedades de Estadística), Montevideo, Uruguay.

[7] Abril, J. C., Abril, M. de las M. and Martínez, C. I. (2008). Edgeworth approximation to the distribution of the autocorrelation coefficient in the presence of unit roots. Pakistan Journal of Statistics, 24 (3), 193-206.

[8] Abril, J.C. and Santillan, M.R. (1998). Approximation to the finite sample distribution of a general estimator of the coefficient in a AR(1) model. Pakistan Journal of Statistics, 14 (1), 11-23.

[9] Barndorff-Nielsen, O and Cox, D. R. (1979). Edgeworth and saddlepoint approximations with statistical applications (with discussion). J. R. Statist. Soc. B, 41, 279-312.

[10] Bhattacharya, R.N. and Rao, R.R. (1976). Normal approximations and asymptotic expansions. John Wiley and Sons. New York.

[11] Cox, D. R. (1948). A note on the asymptotic distribution of the range. Biometrika, 35, 311-15.

[12] Daniels, H. E. (1954). Saddlepoint approximations in Statistics. Ann. Math. Statist., 25, 631-50.

[13] Daniels, H. E. (1956), The approximate distribution of serial correlation coefficients, Biometrika, 43, 169-85.

[14] Durbin, J. (1980a), Approximations for densities of sufficient estimators, Biometrika, 67, 311-33.

[15] Durbin, J. (1980b), The approximate distribution of partial serial correlation coefficients calculated from residuals from regression on Fourier series, Biometrika, 67, 335-49.

[16] Edgeworth, F. Y. (1904). The Law of Errors. Trans. Camb. Phil. Soc., 20, 36 and 113 (with a non - printed Appendix in T. C. P. S but handed over with reprints).

[17] Fowler, R. H. (1936). Statistical Mechanics. Cambridge University Press. Cambridge.

[18] Jeffreys, H. (1948). Theory of Probability. Oxford University Press. Oxford.

[19] Khinchin, A. I. (1949). Mathematical Foundations of Statistical Mechanics. Dover Publications. New York.

[20] Phillips, P. C. B. (1977a). Approximations to some finite sample distributions associated with a first order stochastic difference equation. Econometrica, 45, 46385. 
[21] Phillips, P. C. B., A general theorem in the theory of asymptotic expansions as approximations to the finite sample distributions of econometric estimators. Econometrica, 45 (1977b) 1517-34.

[22] Phillips, P. C. B., Edgeworth and saddlepoint approximations in a first order autoregression. Biometrika,
65 (1978) 91-8.

[23] Stuart, A. y Ord, J. K., Kendall's Advance Theory of Statistics, Vol. 1. Charles Griffin and Company Limited. London, 1987.

[24] Watson, G. N., Theory of Bessel Functions. Cambridge University Press. Cambridge, 1948. 